\title{
A potential signature of eight long non-coding RNAs predicts survival in patients with non-small cell lung cancer
}

\author{
Meng Zhou ${ }^{1,3+}$, Maoni Guo ${ }^{1 \dagger}$, Dongfeng He ${ }^{2 \dagger}$, Xiaojun Wang ${ }^{1}$, Yinqiu Cui ${ }^{3}$, Haixiu Yang ${ }^{1}$, Dapeng Hao ${ }^{\text {* }}$ \\ and Jie Sun ${ }^{1 *}$
}

\begin{abstract}
Background: Accumulated evidence suggests that dysregulated expression of long non-coding RNAs (IncRNAs) may play a critical role in tumorigenesis and prognosis of cancer, indicating the potential utility of IncRNAs as cancer prognostic or diagnostic markers. However, the power of IncRNA signatures in predicting the survival of patients with non-small cell lung cancer (NSCLC) has not yet been investigated.

Methods: We performed an array-based transcriptional analysis of InCRNAs in large patient cohorts with NSCLC by repurposing microarray probes from the gene expression omnibus database. A risk score model was constructed based on the expression data of these eight InCRNAs in the training dataset of NSCLC patients and was subsequently validated in other two independent NSCLC datasets. The biological implications of prognostic IncRNAs were also analyzed using the functional enrichment analysis.

Results: An expression pattern of eight IncRNAs was found to be significantly associated with overall survival (OS) of NSCLC patients in the training dataset. With the eight-IncRNA signature, patients of the training dataset could be classified into high- and low-risk groups with significantly different OS (median survival 1.67 vs. 6.06 years, log-rank test $p=4.33 E-09$ ). The prognostic power of eight-IncRNA signature was further validated in other two non-overlapping independent NSCLC cohorts, demonstrating good reproducibility and robustness of this eight-IncRNA signature in predicting OS of NSCLC patients. Multivariate regression and stratified analysis suggested that the prognostic power of the eight-IncRNA signature was independent of clinical and pathological factors. Functional enrichment analyses revealed potential functional roles of the eight prognostic IncRNAs in tumorigenesis.
\end{abstract}

Conclusions: These findings indicate that the eight-IncRNA signature may be an effective independent prognostic molecular biomarker in the prediction of NSCLC patient survival.

Keywords: Long non-coding RNA, Non-small cell lung cancer, Overall survival, Signature

\section{Background}

Lung cancer is one of the most common human cancers and is the leading cause of cancer-related deaths among both men and women globally [1], accounting for about $27 \%$ of all cancer-related deaths. In China, lung cancer

\footnotetext{
*Correspondence: haodapeng@ems.hrbmu.edu.cn;

suncarajie@hotmail.com

${ }^{\dagger}$ Meng Zhou, Maoni Guo and Dongfeng He contributed equally to this work

${ }^{1}$ College of Bioinformatics Science and Technology, Harbin Medical University, Harbin 150081, People's Republic of China

Full list of author information is available at the end of the article
}

has become the primary cause of cancer-related deaths, and mortality has increased by more than four times during the past three decades [2]. The overall 5-year relative survival rate for lung cancer is low at nearly $15 \%$, which is primarily due to principal detection at late, incurable stages and a paucity of late-stage treatments [3]. Lung cancer is generally divided into two main categories: small cell lung cancer and non-small cell lung cancer (NSCLC) accounting for approximately $80 \%$ of all lung cancers.

Long non-coding RNAs (lncRNAs), a recently discovered subclass of non-coding RNA (ncRNA), are most 
commonly defined as RNA transcripts longer than 200 nucleotides with little coding capacity $[4,5]$. Though the functions of only a limited number of lncRNAs have been well characterized, accumulating evidence has suggested that lncRNAs participate in a wide variety of biological processes, including cell differentiation, organogenesis, chromatin modification, genomic imprinting, dosage compensation, respond to diverse stimuli and so on, by exerting their functions as four archetypes: signals, decoys, guides and scaffolds [6, 7]. IncRNAs can regulate gene expression at the post-transcriptional level via competing endogenous RNA (ceRNA) crosstalk or at the transcriptional level via cis or trans and at the epigenetic regulation level [8-10]. Recently, a number of cancer-related studies have detected many dysregulated IncRNAs associated with tumorigenesis and tumor progression in a variety of cancers [11-13]. Like proteincoding genes and miRNA, some dysregulated lncRNAs play oncogene-like roles. For instance, HOTAIR is an lncRNA that is overexpressed in breast tumors and significantly associated with breast cancer metastasis [14]. Overexpression of lncRNA PCAT-1 is associated with poor prognosis in patients with colorectal cancer (CRC) [15]. Other well-studied lncRNAs, such as MEGS, GAS5, LINOO312 and LinRNA-p21, have instead demonstrated tumor suppressive roles [16, 17]. For example, lncRNA LIN00312, which is significantly down-regulated in nasopharyngeal carcinoma (NPC), was found to be an independent contributor to NPC [18]. These findings suggest that, like protein-coding genes and miRNAs, lncRNAs could serve as diagnostic and prognostic biomarkers. Li et al. [19] measured lncRNA expression in paired tumors and adjacent normal tissues of 119 patients and identified a three-lncRNA signature that could predict the survival of patients with oesophageal squamous cell carcinoma (OSCC). Recent studies have also demonstrated emerging roles of lncRNAs in NSCLC [20]. For example, lncRNA MALAT1 (metastasis-associated lung adenocarcinoma transcript 1) is up-regulated in NSCLC based on evidence from subtractive hybridization of cDNA libraries, and can be used as an independent prognostic marker of patient survival [21]. White and colleagues [22] found 111 differentially expressed lncRNAs between lung tumors and adjacent normal tissues, some of which have been functionally validated to be involved in cellular proliferation in vitro. Nie et al. [23] identified an lncRNA $M V I H$ which is over-expressed in NSCLC tissues compared with adjacent normal tissues. Subsequent studies, integrating custom-designed gene microarray and clinical information, also discovered lncRNA signatures that were significantly associated with the survival of patients with gliolastoma multiforme [24], colorectal cancer [25] and breast cancer [26]. Other recent studies have characterized tens of lncRNAs that were identified to be associated with the presence of certain lung cancer histological subtypes [27, 28]. While the prognostic power of mRNA and miRNA signatures in various cancers is well established, the power of lncRNA signatures in predicting the survival of patients with NSCLC has not yet been investigated.

In the present study, we conducted a comprehensive study of lncRNA expression profiles across 603 NSCLC patients with clinical information by repurposing the previously published NSCLC gene expression profiles, and identified an eight-lncRNA signature associated with survival. A risk score formula was constructed based on the expression data of these eight lncRNAs in the training dataset of NSCLC patients and was further confirmed in another two independent gene expression omnibus (GEO) NSCLC patient cohorts.

\section{Methods}

\section{NSCLC datasets and patient information}

NSCLC microarray datasets, generated with the Affymetrix platform (HG-U133A Plus 2.0), and clinical information were obtained from the GEO database. After removing the patients without available survival information, a total of 603 patients were enrolled in this study (Table 1), including 196 patients from GSE37745 (www. ncbi.nlm.nih.gov/geo/query/acc.cgi?acc $=$ GSE37745) [29], 226 patients from GSE31210 (www.ncbi.nlm.nih. gov/geo/query/acc.cgi?acc $=$ GSE31210) [30] and 181 patients from GSE50081 (www.ncbi.nlm.nih.gov/geo/ query/acc.cgi?acc $=$ GSE50081) [31]. More detailed clinical information of all 603 NSCLC patients included in this study can be found in Table 1 .

\section{Microarray processing and IncRNA profile mining}

All the microarray raw data (.CEL files) of three NSCLC cohorts were obtained from the GEO database and processed using the robust multichip average (RMA) algorithm for background adjustment [32, 33]. The Affymetrix GeneChip probe-level data was log-2-scale transformed and standardized by transforming the expression data into having a mean of 0 and a standard deviation (SD) of 1 . The NetAffx probe set sequences for Affymetrix HG-U133 Plus 2.0 were downloaded from the Affymetrix website (http://www.affymetrix.com). LncRNA expression data from the Affymetrix-based expression profiling of NSCLC cohorts were obtained by repurposing microarray probes based on the sequences of the probe sets and the annotations of lncRNAs in GENCODE (http://www.gencodegenes.org/) (GRCh38, release 21) [34], as previous described [35]. By keeping probes that were uniquely mapped to the genomic coordinate of lncRNAs derived from GENCODE, 3,521 probes (or 
Table 1 Clinical features of all 603 NSCLC patients included in this study

\begin{tabular}{|c|c|c|c|}
\hline Features & GSE37745 $(n=196)$ & GSE31210 $(n=226)$ & GSE50081 $(n=181)$ \\
\hline \multicolumn{4}{|l|}{ Age (years), no (\%) } \\
\hline$\leq 65$ & $94(48.0)$ & $176(77.9)$ & $59(32.6)$ \\
\hline$>65$ & $102(52.0)$ & $50(22.1)$ & $122(67.4)$ \\
\hline \multicolumn{4}{|l|}{ Gender, no (\%) } \\
\hline Male & $107(55.0)$ & $105(46.5)$ & $98(54.1)$ \\
\hline Female & $89(45.0)$ & $121(53.5)$ & $83(45.9)$ \\
\hline \multicolumn{4}{|l|}{ Vital status (\%) } \\
\hline Alive & $52(26.5)$ & $191(84.5)$ & $106(58.6)$ \\
\hline Dead & $144(73.5)$ & $35(15.5)$ & $75(41.4)$ \\
\hline \multicolumn{4}{|l|}{ Disease stage, no (\%) } \\
\hline I & $130(66.0)$ & $168(74.3)$ & $127(70.2)$ \\
\hline$\|$ & $35(18.0)$ & $58(25.7)$ & $54(29.8)$ \\
\hline III & $27(14.0)$ & & \\
\hline IV & $4(2.0)$ & & \\
\hline \multicolumn{4}{|l|}{ Smoking status } \\
\hline Never-smoker & & $115(50.9)$ & $24(13.3)$ \\
\hline Ever-smoker & & $111(49.1)$ & 79 (43.6) \\
\hline Current & & & $54(29.8)$ \\
\hline Undetermined & & & $21(11.6)$ \\
\hline \multicolumn{4}{|l|}{ Histology } \\
\hline Adenocarcinoma & $106(54.0)$ & 226 & $128(70.7)$ \\
\hline Large cell carcinoma & $24(12.0)$ & & $7(3.9)$ \\
\hline Squamous cell carcinoma & $66(34.0)$ & & $43(23.9)$ \\
\hline
\end{tabular}

probe sets) and 2,313 corresponding lncRNA genes were obtained. Multiple probes (or probe sets) mapping to the same gene were integrated by using the arithmetic mean of the values of multiple probes (or probe sets) to generate a single gene expression value (on the $\log 2$ scale).

\section{Statistical analysis}

A univariable Cox regression analysis was performed to evaluate the relationship between the continuous expression level of each lncRNA and patients' overall survival (OS) in the training dataset. Only those lncRNAs with a $p$ value of $<0.005$ were considered statistically significant. To construct a predictive model, each of the selected lncRNA genes was analyzed using a multivariable Cox regression model in the training dataset, with OS as the dependent variable and other clinical information as the covariables. A risk score was then computed as follows:

$$
\operatorname{Risk} \operatorname{Score}(R S)=\sum_{i=1}^{N}\left(\operatorname{Exp}_{i} * \operatorname{Coe}_{i}\right)
$$

where $N$ is the number of prognostic lncRNA genes, $\operatorname{Exp}_{i}$ is the expression value of $\ln c R N A_{i}$, and $C_{0} e_{i}$ is the estimated regression coefficient of $\ln c R N A_{i}$ in the multivariable Cox regression analysis. This risk score model was established by taking into account the power of each of the prognostic lncRNA genes.

Using the median risk score in the training dataset as a cutoff value, NSCLC patients in each dataset were divided into high- and low-risk groups. Kaplan-Meier survival analyses were performed to test the equality for survival distributions in different groups for each NSCLC cohort, and statistical significance was assessed using the two-sided log-rank test. Additionally, a multivariable Cox regression analysis and data stratification analysis were performed to test whether the risk score was independent of other clinical features within the available data. The time-dependent receiver operating characteristic (ROC) curve was also used to compare the sensitivity and specificity of the survival prediction of the lncRNA expression-based risk score in the training dataset. Area under the curve (AUC) value was calculated from the ROC curve. All analyses were performed using $\mathrm{R}$ software and Bio-conductor. Significance was defined as $\mathrm{p}<0.05$.

Bioinformatics analysis of IncRNA gene function prediction The co-expressed relationships between the prognostic lncRNAs and protein-coding genes were computed using Pearson correlation coefficients. Gene ontology 
(GO) and Kyoto encyclopedia of genes and genomes (KEGG) enrichment analyses of the co-expressed protein-coding genes with prognostic lncRNAs were performed to predict the biological function of prognostic lncRNAs using the DAVID Bioinformatics Tool (version 6.7), which is a commonly used functional annotation tool that can assess over-representation of functional categories among a gene set of interest [37]. Enrichment analysis was carried out using the functional annotation chart and functional annotation clustering options, and was limited to KEGG pathways and GO terms in the "Biological Process" categories. Functional annotation with $p$ value of $<0.05$ and an enrichment score of $>2$ were considered significant.

\section{Results}

Derivation of an eight-IncRNA prognostic signature from the training dataset

The NSCLC patient cohort from GSE37745 $(\mathrm{n}=196)$, including the relatively large patient sample size and relatively overall clinical information, was selected as training dataset to explore the association between IncRNA expression and OS of NSCLC patients. We first conducted a univariate Cox proportional hazards regression analysis of the lncRNA expression data with OS as the dependent variable, and identified a set of eight lncRNAs as prognostic lncRNAs which were significantly correlated with patients' OS ( $p$ value of $<0.005$ ). Table 2 shows a list of these eight prognostic lncRNAs along with important variable information. Of the eight lncRNAs, the higher expression level of IncRNA RP11-21L23.2, GPR158-AS1, RP11-701P16.5 and RP11-379F4.4 was associated with shorter OS (coefficient $>0$ ), and the higher expression levels of the remaining four lncRNAs (CTD-2358C21.4, RP11-94L15.2, KCNK15-AS1 and AC104134.2) were associated with longer OS (coefficient $<0$ ). Then we further examined whether these eight prognostic lncRNAs are differentially expressed between cancer and normal lung tissue. The lncRNA differential expression analysis was performed for GSE18842 dataset (including 46 tumor and 45 normal lung tissue specimens) (http://www.ncbi.nlm.nih.gov/geo/query/acc. cgi?acc $=$ GSE18842) [38] obtained from GEO database. We found that five of eight prognostic lncRNAs showed significant expression differences between tumor and normal lung tissue (Mann-Whitney $\mathrm{U}$ test $\mathrm{p}<0.05$ ) (Additional file 1: Figure S1), demonstrating that these selected prognostic lncRNAs are associated with lung cancer.

\section{An eight-IncRNA signature predicts survival of NSCLC patients in the training dataset}

To investigate whether the eight-lncRNA signature could provide an accurate prediction of OS in NSCLC patients, the expression data of these eight lncRNAs and other clinical features were fitted into a multivariable Cox regression model as covariates of the training dataset. A risk score was generated for each patient in the training dataset according to the risk-score model (see "Methods") as follows: Risk score $=(0.306 \times$ expression value of RP11-21L23.2) $+(-0.314 \times$ expression value of $C T D-2358 C 21.4)+(-0.252 \times$ expression value of $R P 11-94 L 15.2)+(0.288 \times$ expression value of GPR158-AS1) + (-0.271 $\times$ expression value of KCNK15$A S 1)+(-0.299 \times$ expressionvalueof $A C 104134.2)+(0.284 \times$ expression value of $R P 11-701 P 16.5)+(0.321 \times$ expression value of RP11-379F4.4). To evaluate how well the risk score predicts the 5-year survival, the various cutoff values were evaluated using time-dependent ROC curve (Figure 1a) which is commonly used for revealing the predictive accuracy of a model $[39,40]$. In the training dataset, AUC for the eight-lncRNA signature prognostic model was 0.78 at an OS of 5 years, demonstrating the better performance for survival prediction of the lncRNA expression-based risk score in the training dataset. All patients in the training dataset were then ranked according to their risk score, and divided into either the

Table 2 Eight IncRNAs significantly associated with the overall survival of NSCLC patients in the training set $(\mathbf{n}=196)$

\begin{tabular}{llllrr}
\hline Ensembl id & Gene symbol & Chromosomal position & P value $^{\mathbf{a}}$ & Hazard ratio $^{\mathbf{a}}$ & Coefficient $^{\mathbf{a}}$ \\
\hline ENSG00000261578.1 & RP11-21L23.2 & Chr11: 76,800,364-76,804,555(+) & $2.57 \mathrm{E}-05$ & 1.374 & 0.318 \\
ENSG00000261731.2 & CTD-2358C21.4 & Chr16:31,709,113-31,711,984(-) & $2.22 \mathrm{E}-04$ & 0.725 & -0.322 \\
ENSG00000264198.2 & RP11-94L15.2 & Chr17:39,757,715-39,763,836(-) & $2.88 \mathrm{E}-04$ & 0.738 & -0.303 \\
ENSG00000233642.1 & GPR158-AS1 & Chr10: 25,158,072-25,176,276(-) & $4.52 \mathrm{E}-04$ & 1.334 & 0.288 \\
ENSG00000244558.3 & KCNK15-AS1 & Chr20: 44,694,892-44,746,021(-) & $1.27 \mathrm{E}-03$ & 0.750 & -0.287 \\
ENSG00000225420.1 & AC104134.2 & Chr2: 88,538,720-88,575,610(+) & $1.85 \mathrm{E}-03$ & 0.760 & -0.274 \\
ENSG00000251230.3 & RP11-701P16.5 & Chr4: 184,844,585-184,855,751(-) & $2.01 \mathrm{E}-03$ & 1.301 & 0.263 \\
ENSG00000240207.4 & RP11-379F4.4 & Chr3: 158,732,263-158,784,070(+) & $2.80 \mathrm{E}-03$ & 1.433 & 0.360 \\
\hline
\end{tabular}

${ }^{a}$ Derived from the univariable Cox regression analysis in the training set. 


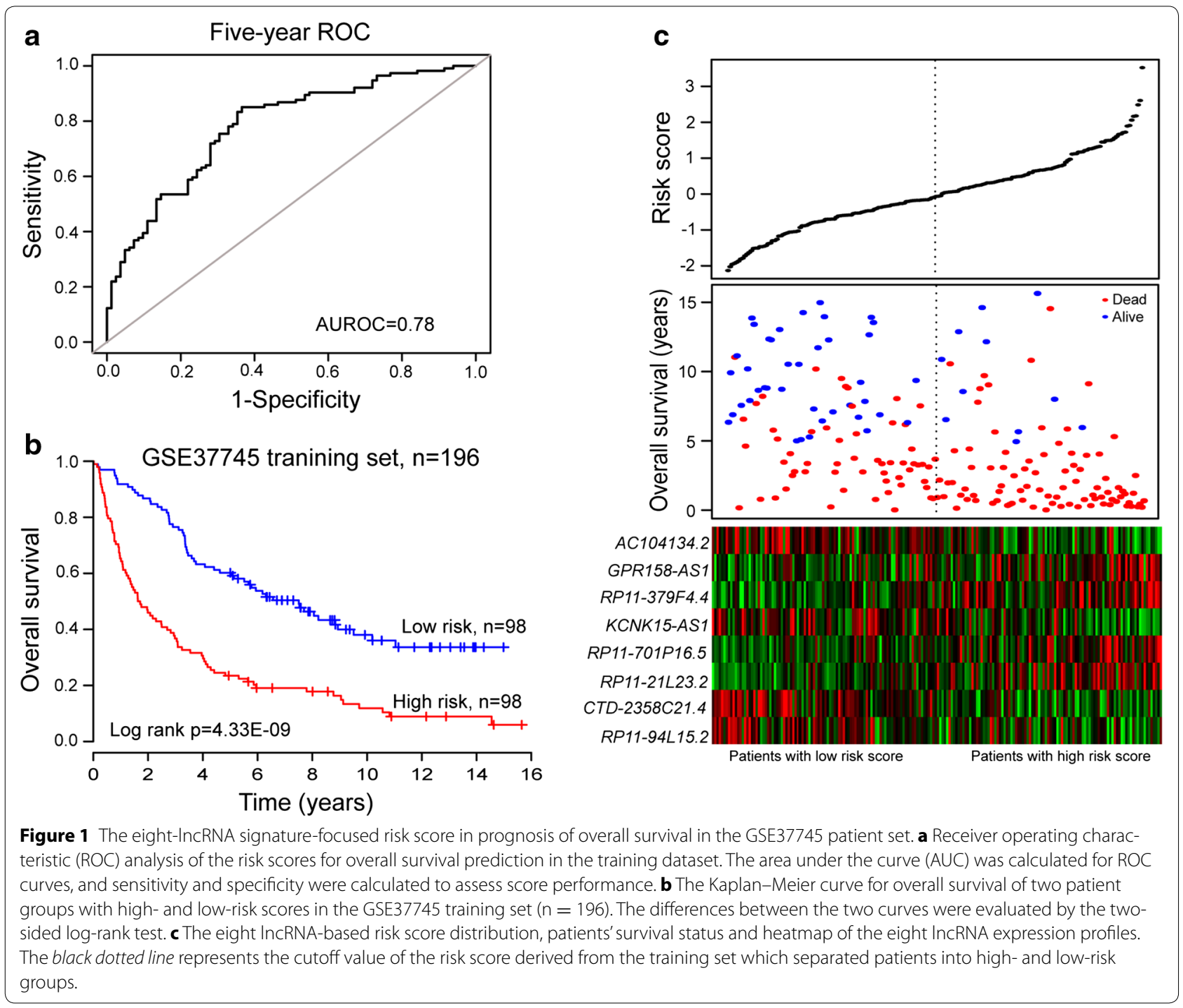

high- or low-risk group using the median risk score as the cutoff point. According to this cutoff value, patients were divided into either a high-risk group $(n=98)$ or a low-risk group $(\mathrm{n}=98)$. Patients in the high-risk group had a significantly shorter OS than those in the lowrisk group (median OS 1.67 vs. 6.06 years, log-rank test $\mathrm{p}=4.33 \mathrm{E}-09$ ). Kaplan-Meier curves for the high- and low-risk groups in the training dataset $(n=196)$ are shown in Figure 1b. In detail, OS rates of patients in the high-risk group were $30.6 \%$ at 4 years, $19.1 \%$ at 6 years, $17.8 \%$ at 8 years and $11.9 \%$ at 10 years, versus $63.3,53.8$, 46.3 and $38 \%$ in the low-risk group, respectively.

A significant association between the eight-lncRNA signature risk score and OS was observed in the univariable Cox regression model (Table 3 ). The hazard ratios of the eight-lncRNA signature risk score of the high-risk group versus that of the low-risk group for OS was 2.641 [p < 0.001; 95\% confidence interval (CI) 1.887-3.697; Table 3].

The distribution of risk score, survival status and prognostic lncRNA expression in 196 patients of the training dataset are shown in Figure 1c. Of these eight prognostic lncRNAs, the high expression level of lncRNA RP1121L23.2, GPR158-AS1, RP11-701P16.5 and RP11-379F4.4 was associated with high risk, while the remaining four lncRNAs (CTD-2358C21.4, RP11-94L15.2, KCNK15-AS1 and $A C 104134.2)$ were shown to be protective. NSCLC patients with high prognostic scores tended to express high-risk lncRNAs, whereas those with low prognostic scores tended to express protective lncRNAs. Moreover, more deaths were noted for NSCLC patients with highrisk scores than for those with low-risk scores. 
Table 3 Univariable and multivariable Cox regression analysis of the IncRNA signature and overall survival of NSCLC patients in the training and two independent cohorts

\begin{tabular}{|c|c|c|c|c|c|c|}
\hline \multirow[t]{2}{*}{ Variables } & \multicolumn{3}{|c|}{ Univariable analysis } & \multicolumn{3}{|c|}{ Multivariable analysis } \\
\hline & HR & $95 \% \mathrm{Cl}$ of $\mathrm{HR}$ & $P$ value & HR & $95 \% \mathrm{Cl}$ of $\mathrm{HR}$ & $P$ value \\
\hline \multicolumn{7}{|c|}{ GSE37745 training set, $\mathrm{n}=196$} \\
\hline \multicolumn{7}{|c|}{ Eight-IncRNA risk score } \\
\hline Low risk/high risk & 2.641 & $1.887-3.697$ & $<0.001$ & 2.761 & $1.934-3.942$ & $<0.001$ \\
\hline \multicolumn{7}{|l|}{ Age } \\
\hline$\leq 65 />65$ & 1.355 & $0.977-1.878$ & 0.069 & 1.427 & $1.024-1.986$ & 0.035 \\
\hline \multicolumn{7}{|l|}{ Gender } \\
\hline Female/male & 1.096 & $0.789-1.523$ & 0.585 & 0.913 & $0.640-1.303$ & 0.616 \\
\hline \multicolumn{7}{|l|}{ Stage } \\
\hline 1 & 1 (reference) & & & 1 (reference) & & \\
\hline$\|$ & 1.220 & $0.793-1.875$ & 0.366 & 1.169 & $0.758-1.802$ & 0.479 \\
\hline III & 1.864 & $1.187-2.928$ & 0.007 & 1.656 & $1.052-2.608$ & 0.029 \\
\hline IV & 1.313 & $0.415-4.152$ & 0.643 & 1.566 & $0.488-5.022$ & 0.451 \\
\hline \multicolumn{7}{|l|}{ Subtype } \\
\hline Adenocarcinoma & 1 (reference) & & & 1 (reference) & & \\
\hline $\begin{array}{l}\text { Large cell carci- } \\
\text { noma }\end{array}$ & 0.891 & $0.520-1.528$ & 0.675 & 0.782 & $0.449-1.360$ & 0.383 \\
\hline $\begin{array}{l}\text { Squamous cell } \\
\text { carcinoma }\end{array}$ & 1.257 & $0.883-1.791$ & 0.205 & 0.920 & $0.623-1.359$ & 0.676 \\
\hline \multicolumn{7}{|c|}{ GSE31210 testing set, $n=226$} \\
\hline \multicolumn{7}{|c|}{ Eight-IncRNA risk score } \\
\hline Low risk/High risk & 3.067 & $1.471-6.395$ & 0.003 & 2.643 & $1.263-5.528$ & 0.010 \\
\hline \multicolumn{7}{|l|}{ Age } \\
\hline$\leq 65 />65$ & 2.584 & $1.313-5.083$ & 0.006 & 3.685 & $1.800-7.544$ & $<0.001$ \\
\hline \multicolumn{7}{|l|}{ Gender } \\
\hline Female/male & 1.519 & $0.780-2.955$ & 0.219 & 1.143 & $0.402-3.246$ & 0.802 \\
\hline \multicolumn{7}{|l|}{ Smoking status } \\
\hline No/Yes & 1.637 & $0.837-3.201$ & 0.15 & 1.388 & $0.482-3.996$ & 0.544 \\
\hline \multicolumn{7}{|l|}{ Stage } \\
\hline$|/| \mid$ & 4.232 & $2.175-8.236$ & $<0.001$ & 4.363 & $2.161-8.811$ & $<0.001$ \\
\hline \multicolumn{7}{|c|}{ GSE50081 testing set, $\mathrm{n}=181$} \\
\hline \multicolumn{7}{|c|}{ Eight-IncRNA risk score } \\
\hline Low risk/high risk & 1.795 & $1.127-2.859$ & 0.014 & 1.752 & $1.014-3.026$ & 0.044 \\
\hline \multicolumn{7}{|l|}{ Age } \\
\hline$\leq 65 />65$ & 1.559 & $0.932-2.608$ & 0.090 & 1.316 & $0.752-2.303$ & 0.336 \\
\hline \multicolumn{7}{|l|}{ Gender } \\
\hline Female/male & 1.934 & $1.190-3.143$ & 0.008 & 1.743 & $1.011-3.005$ & 0.046 \\
\hline \multicolumn{7}{|l|}{ Smoking status } \\
\hline No/Yes & 1.387 & $0.659-2.916$ & 0.389 & 1.054 & $0.476-2.333$ & 0.897 \\
\hline \multicolumn{7}{|l|}{ Stage } \\
\hline$|/| \mid$ & 1.689 & $1.049-2.718$ & 0.031 & 2.359 & $1.379-4.034$ & 0.002 \\
\hline \multicolumn{7}{|l|}{ Subtype } \\
\hline Adenocarcinoma & 1 (reference) & & & 1 (reference) & & \\
\hline $\begin{array}{l}\text { Large cell carci- } \\
\text { noma }\end{array}$ & 1.326 & $0.479-3.671$ & 0.587 & 1.094 & $0.376-3.184$ & 0.870 \\
\hline $\begin{array}{l}\text { Squamous cell } \\
\text { carcinoma }\end{array}$ & 0.791 & $0.456-1.371$ & 0.403 & 0.479 & $0.241-0.952$ & 0.036 \\
\hline
\end{tabular}




\section{Validation of the eight-IncRNA signature for survival prediction in the testing GSE31210 dataset}

To validate the prognostic power of the eight-lncRNA signature for survival prediction, the constructed expression-defined lncRNA prognostic model was also evaluated in the testing GSE31210 dataset. The same prognostic risk score model obtained from the training dataset was used to calculate the eight-lncRNA signature-based risk scores for 226 patients in the entire GSE31210 dataset. The cutoff value of the risk score derived from the training dataset without re-estimating parameters was used for the testing dataset to classify the patients into either a high-risk group $(n=111)$ or a low-risk group $(\mathrm{n}=115)$. Patients with high-risk scores exhibited poorer OS than those with low-risk scores (median OS 4.45 vs. 5.08 years, log-rank test $\mathrm{p}=1.65 \mathrm{E}-03)$. Kaplan-Meier curves for the high- and low-risk groups in the testing dataset are shown in Figure $2 \mathrm{a}$. The OS rate of patients in the high-risk group was $91.7 \%$ at 2 years and $78.7 \%$ at 4 years, versus 97.4 and $91.5 \%$ in the low-risk group, respectively. A significant association between the eight-lncRNA signature risk score and OS in the univariable Cox regression model was observed. The hazard ratios of the eightlncRNA signature risk scores of the high-risk group versus the low-risk group for OS was 3.067 ( $\mathrm{p}=0.003$; 95\% CI 1.471-6.395; Table 3).

The distribution of patient lncRNA risk score, survival status and prognostic lncRNA expression in 226 patients of the GSE31210 dataset are shown in Figure 2b, revealing the similar results observed in the GSE37745 training dataset.

\section{Further validation of the eight-IncRNA signature in another independent dataset}

To investigate the reproducibility of the eight-lncRNA signature in predicting OS of NSCLC patients, the prognostic power of the eight-lncRNA signature for prediction of survival was further validated in another independent NSCLC cohort of 181 patients whose expression and survival data were obtained from GEO GSE50081. The clinical feature of this independent NSCLC cohort is shown in Table 1. Patients in this independent NSCLC cohort were classified into either a high-risk group $(n=90)$ or a low-risk group $(n=91)$ according to the cutoff value of risk scores obtained from the training dataset. The median OS of the high-risk group for the GSE50081 dataset is 4.29 years, whereas that of the low-risk group is 4.99 years (log-rank test $\mathrm{p}=1.26 \mathrm{E}-02$ ). KaplanMeier curves for the high- and low-risk groups within the independent GSE50081 cohort is shown in Figure 2c. Further univariable Cox regression analysis revealed that the high-risk scores of eight-lncRNA signature was significantly associated with lower OS of patients in GSE50081 dataset $(\mathrm{p}=1.40 \mathrm{E}-02$; HR $=1.795,95 \% \mathrm{CI}$ 1.127-2.859; Table 3). Figure $2 \mathrm{~d}$ shows the distribution of patient risk scores, the survival status and prognostic lncRNA expression in the independent GSE50081 NSCLC cohort, ranked according to the prognostic risk score values for the eight-lncRNA signature, which were similar to those observed in the training and GSE31210 datasets.

\section{Survival prediction by the eight-IncRNA signature is independent of clinical features}

To assess whether the prognostic power of the eightlncRNA signature for prediction of survival was independent of other clinical features, multivariable Cox regression analysis was performed using the lncRNA signature-based risk score and other clinical features, including age, gender, smoking status, tumor stage and subtype, which were used as covariates. The results of multivariable Cox regression analysis from three NSCLC patients datasets showed that the prognostic power of the eight-lncRNA signature risk score (highrisk group vs. low-risk group, $\mathrm{HR}=2.761,95 \% \mathrm{CI}$ 1.934-3.942, $\mathrm{p}<0.001$ for GSE37745; HR $=2.643$, 95\% CI 1.263-5.528, $\mathrm{p}=0.01$ for GSE31210; $\mathrm{HR}=1.752$, 95\% CI 1.014-3.026, $\mathrm{p}=0.044$ for GSE50081) for prediction of survival was indeed independent of these clinical features (Table 3). We also found that the two clinical factors, age and stage, also affected overall survival of patients. So, a data stratification analysis was performed according to age and stage. The three GEO datasets (GSE37745, GSE31210 and GSE50081), which included a total of 603 patients, were stratified by age into either a younger stratum (age $\leq 65)$ or an elder stratum (age $>65$ ). The results of stratified analysis showed effective prognostic power in both the younger and elder patient groups. The eight-lncRNA signature could classify patients within each age stratum into either high- or low-risk groups with significantly different OS (log-rank test $\mathrm{p}=4.46 \mathrm{E}-05$ for the younger patient group and $\mathrm{p}=6.61 \mathrm{E}-06$ for the elder patient group) (Figure 3a, b), which suggested that the prognostic power of the eight-lncRNA signature was also age-independent. Then the patients of early (stage I and II) and late (III and IV) stage for GSE37745 dataset were grouped into two separate groups. The stratified analysis was further performed in early and late stage patients to evaluate whether the eight-lncRNA signature could predict survival of patients for different clinical stage. The log-rank test of early stage patients showed that within stage I and II, the eight-lncRNA signature could further subdivide them into either a high-risk group with shorter survival or a low-risk group with longer survival (median 
OS 2.03 vs. 8.05 years, log-rank test $\mathrm{p}=7.81 \mathrm{E}-09$ ) (Figure 3c). Difference for OS between high-risk group $(\mathrm{n}=18)$ and low-risk group $(\mathrm{n}=13)$ also was observed for late stage patients (median OS 0.975 vs. 3.367 years) (Figure $3 \mathrm{~d}$ ), although the log-rank $\mathrm{p}$ value is 0.253 which was above the 0.05 significance level.

\section{Functional characterization of the eight prognostic IncRNAs}

To further investigate the potential biological roles involving the eight prognostic lncRNAs, the co-expressed relationships between the expression of eight lncRNAs and those of the protein-coding genes were computed using Pearson correlation coefficients in the GSE37745 dataset of 196 patients. The expression of 679 proteincoding genes were highly correlated with that of at least one of the eight signature lncRNAs (Pearson correlation coefficient $>0.40$ ). GO and KEGG pathway function enrichment analysis for these co-expressed protein-coding genes was then performed, using the whole human genome as the background. The results showed that four genes (GATA6, CRISPLD2, CFTR2 and CLPTM1L) have been proven to be involved in lung cancer. GO functional annotation suggested that 679 protein-coding genes were significantly enriched in $28 \mathrm{GO}$ terms (Figure 4a). These significant GO terms were organized into an interaction network with similar functions using the Enrichment Map [41] plugin in Cytoscape [42]. Several clusters of functionally related GO terms were observed including organ development and cell proliferation and immune, response to stimulus, catabolic and metabolic process (Figure 4b). Taken together, these results implied that
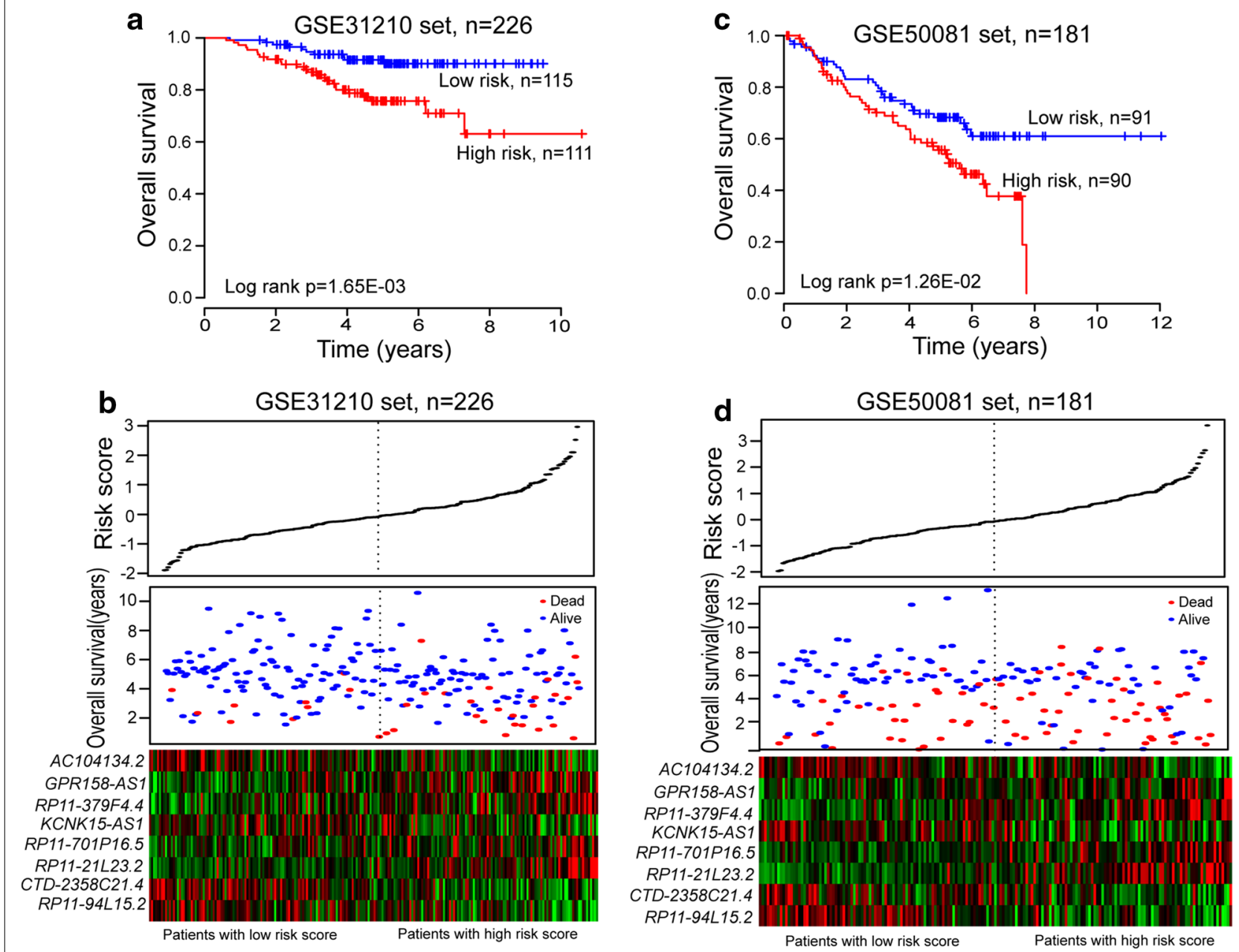

Figure 2 The eight-IncRNA signature-focused risk score in prognosis of overall survival in additional validation datasets. a Kaplan-Meier survival curves were plotted for GSE31210 ( $n=226)$. b The eight IncRNA-based risk score distribution, patients' survival status and heatmap of the eight IncRNA expression profiles were analyzed in the GSE31210. c Kaplan-Meier survival curves were plotted for GSE50081 ( $n=181)$. d The eight IncRNA-based risk score distribution, patients' survival status and heatmap of the eight IncRNA expression profiles were analyzed in the GSE50081. 
the eight lncRNAs might be involved in tumorigenesis through interacting with protein-coding genes that affect the tissue/organ development and other important biological processes.

\section{Discussion}

During the past few decades, considerable efforts have been made toward the development of gene-expression-based diagnostic and prognostic biomarkers for lung cancer at the protein-coding genes and miRNAs levels $[43,44]$. However, accumulating evidence suggested that lncRNA are involved in oncogenic and tumor suppressive pathways have opened the door for this new biomarker. Transcriptional profiling analyses have discovered a number of tissue-specific lncRNAs in normal tissues and dysregulated lncRNAs in a variety of human cancers [11, 45], and highly aberrant expression of dysregulated lncRNAs is associated with tumorigenesis [17]. Furthermore, these dysregulated lncRNAs have already shown great potential as novel molecular biomarkers for diagnosis, prognosis and treatment of cancer. More recently, several studies conducted arraybased transcriptional analyses of lncRNAs and functionally characterized cancer subtype-associated lncRNAs in breast cancer and lung cancer, proposing a novel clinical implication for lncRNAs as valuable biomarkers for prediction of response to treatment as well as patient outcome $[27,46]$. Compared to protein-coding genes, the advantage of lncRNAs as molecular biomarkers is that lncRNA expression is more closely associated with its biological function and tumor status [16, 47]. However, to date, expression profile-based prognostic lncRNA signature for prediction of survival of NSCLC patients has not been investigated.

Recently, several studies have reported that lncRNA expression profiles can be obtained from publicly available, custom-designed DNA microarrays by re-annotating the array probes $[19,25,26,35,47]$. In this study, microarray probe re-annotation was used to repurpose the publicly available human Affymetrix microarray data (HG-U133 Plus 2.0) and subsequently obtain lncRNA expression profiles of 603 NSCLC patients from GEO. To identify lncRNAs with prognostic value in NSCLC, survival analysis was performed by integrating lncRNA expression profiles and clinical information in a large cohort of NSCLC patients. An expression pattern of eight lncRNAs was found to be significantly associated with OS of NSCLC patients in the GSE37745 training dataset. Further ROC analysis demonstrated good performance for predicting 5-year OS. A prognostic risk score model was developed based on the expression data of these eight lncRNAs and weighted by the estimated regression coefficients from multivariable Cox regression analysis. With this eight-lncRNA signature, patients in the training dataset with high-risk scores tended to have lower OS than those with low-risk scores. The separation between survival curves for high- and low-risk patients of the training dataset used for model development was observed. A previous simulation study revealed that a prognostic model can also be developed that is significantly associated with survival time in the training dataset when using completely random gene expression profiles [48]. To evaluate the robustness and reproducibility of the prognostic power of the eight-lncRNA signature, it was also tested in the non-overlapping two other independent NSCLC patient cohorts (GSE31210 and GSE50081) using the same model and criteria as those from the training dataset. In these tests, the prognostic power was also strong, indicating that the eight-lncRNA signature demonstrated good reproducibility and robustness for the NSCLC patients.

Several studies have observed different clinical characteristics and survival time among different age groups of NSCLC patients [49-51]. Multivariable Cox regression analysis was thus used to assess the independence of the eight-lncRNA signature in predicting OS. With age, gender, smoking status, stage and subtype as covariables in the regression analysis, risk score of the eight-lncRNA signature was found to have maintained an independent correlation with OS. In the stratified analysis, the eightlncRNA signature showed prognostic power for different age groups, in which patients belonging to the same age group could be classified into high- and low-risk groups with significantly different survival prospects, indicating that the prognostic value of the eight-lncRNA signature was independent of age of the NSCLC patients. In lung cancer, clinicopathological parameters like tumor histology, staging and localization of metastases determine patients' outcome [52]. Since tumor stage and subtype data was only available for the GSE37745 patient dataset, multivariate Cox regression analysis and stratified analysis were performed to assess the stage- and subtypeindependence of prognostic power of the eight-lncRNA signature. The eight-lncRNA signature was indeed found to be stage-dependent in NSCLC patients, and its prognostic power was significant in stage I and II patients, in which all patients in stage I and II could be separated into high- and low-risk groups with significantly different survival. However, the eight-lncRNA signature achieved a p value of 0.253 for OS prediction of late stage patients, which was above the 0.05 significance level, suggesting that patients with early stage cancer may benefit significantly from this eight-lncRNA prognostic signature. Further multivariate Cox regression analysis testing tumor 

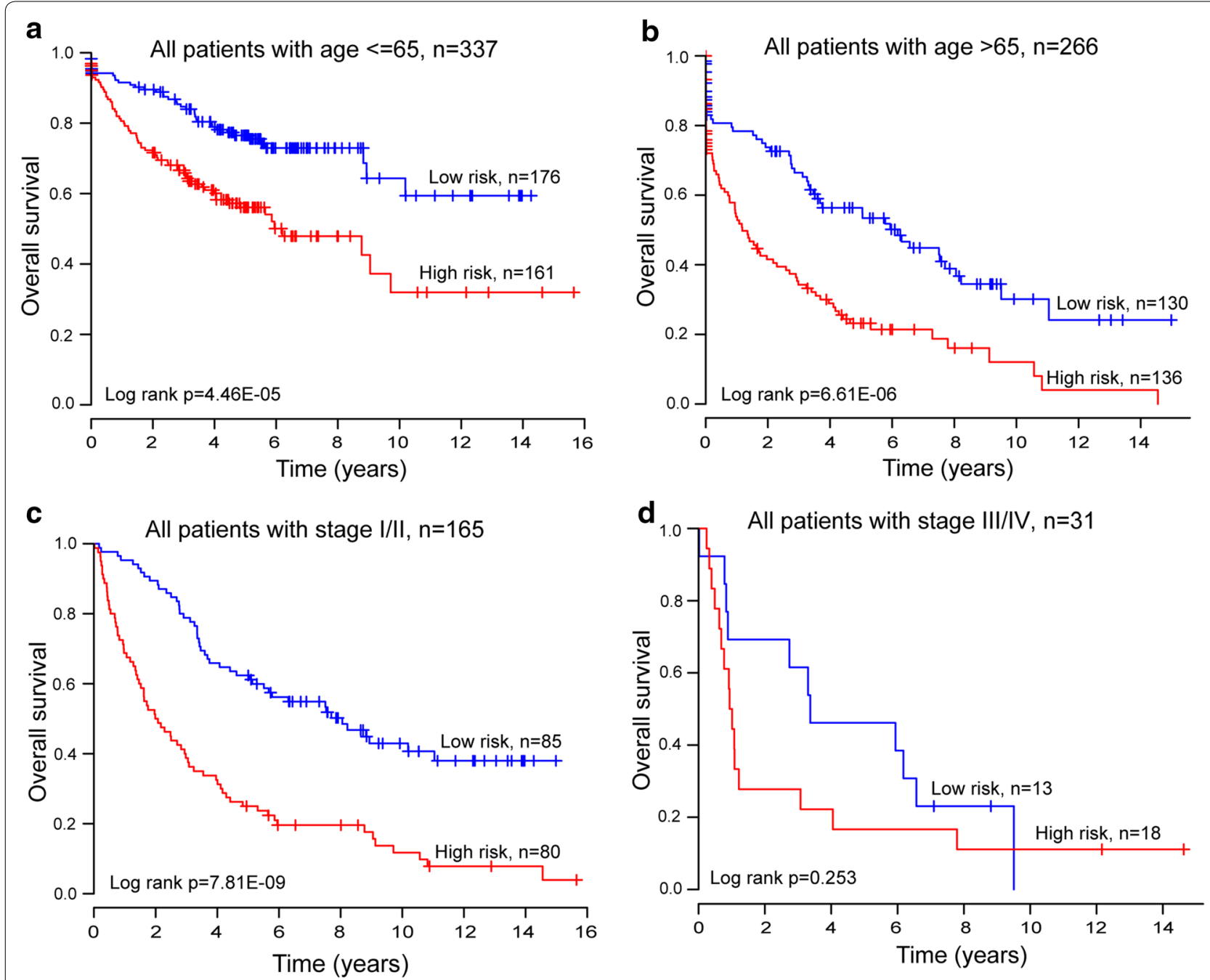

Figure 3 Survival analyses of all patients with available age or tumor stage information using the eight-IncRNA signature. a Kaplan-Meier survival curves for younger patients with NSCLC (age $\leq 65, n=337$ ). b Kaplan-Meier survival curves for elder patients with NSCLC (age $>65, n=226$ ). c Survival prediction in early stage patients: Kaplan-Meier survival curves for all patients with stage I and II $(n=165)$. d Survival prediction in late stage patients: Kaplan-Meier survival curves for all patients with stage III and IV $(n=31)$

subtype-independence suggested that prognostic power of the eight-lncRNA signature is independent of tumor subtype. Taken together, these results suggest that the prognostic power of the eight-lncRNA signature for predicting OS of NSCLC patients is independent of other clinical features except for stage.

Tens of thousands of IncRNAs have been identified and predicted by large-scale transcriptome analysis in humans [53]. However, the functions of only a few lncRNAs have been well characterized, so no thorough functional annotation data is available for the eight prognostic lncRNAs in the current literature. Recent bioinformatics studies have suggested that the function of lncRNAs could effectively be predicted with the inclusion of different kinds of biological data. To increase our understanding of the biological roles of the eight prognostic lncRNAs in NSCLC, functional enrichment analysis was performed for 679 proteincoding genes co-expressed with the eight prognostic lncRNAs at the GO and KEGG pathway level. The biological processes most highly associated with the genes were organ development, cell proliferation and immune, response to stimulus, catabolic and metabolic process. In particular, several co-expressed protein-coding genes with eight prognostic RNAs were proven to participate in the NSCLC pathway. These results implied important functional roles of the eight prognostic lncRNAs in tumorigenesis. 


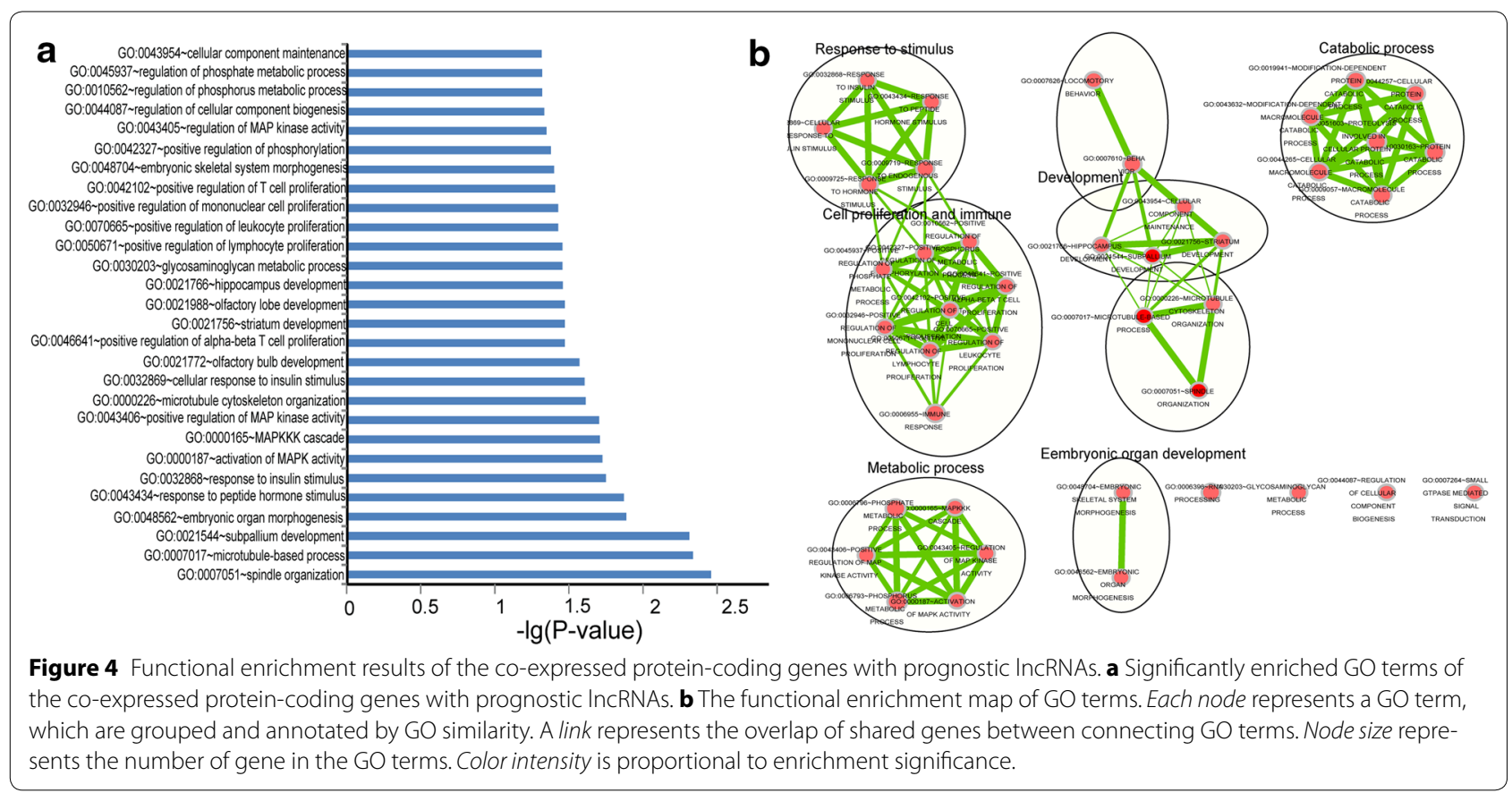

Due to the restriction of available data, gene expression profiles of only 2,313 of the tens-of-thousands of known and predicted lncRNAs were obtained. However, the prognostic power of the eight-lncRNA signature uncovered in this study for predicting OS consistently observed in multiple independent datasets. Moreover, the incompleteness and low coverage of available lncRNA-related datasets are common when by studying lncRNA-disease associations. Although the functions of these eight lncRNAs have been inferred by bioinformatics analysis, the biological roles of these eight lncRNAs in tumorigenesis are still not clear and should be investigated in further experimental studies. With the rapid increase of IncRNA-related studies, more comprehensive lncRNA will become available, and lncRNA biomarker development for clinical prognostic evaluation of NSCLC should increase.

\section{Conclusions}

In summary, by examining lncRNA expression profiles of patients with NSCLC, our study identified eight lncRNAs associated with overall survival of NSCLC patients. A prognostic lncRNA signature was developed based on the expression patterns of these eight lncRNAs in the training dataset to predict the overall survival, and subsequently was validated in other two independent datasets. Further analysis demonstrated that the prognostic power of the eight-lncRNA signature for prediction of survival was independent of other clinical features. Our results suggested that the eightlncRNA signature may be an effective independent prognostic molecular biomarker in the prediction of NSCLC patient survival.

\section{Additional file}

Additional file 1: Figure S1. The boxplot of expression level of eight prognostic IncRNAs in lung cancer and control samples.

\section{Abbreviations}

AUC: area under the ROC curve; CeRNA: competing endogenous RNA; Cl: confidence interval; CRC: colorectal cancer; GEO: gene expression omnibus; GO: gene ontology; HR: hazard ratio; KEGG: Kyoto encyclopedia of genes and genomes; LncRNAs: long non-coding RNAs; MALAT1: metastasis-associated lung adenocarcinoma transcript 1; NPC: nasopharyngeal carcinoma; NSCLC: non-small cell lung cancer; OS: overall survival; OSCC: oesophageal squamous cell carcinoma; RMA: robust multichip average; ROC: receiver operating characteristic; SD: standard deviation.

\section{Authors' contributions}

JS and DPH conceived and designed the experiments. MZ, MNG, DFH, XJW YQC and HXY analyzed data. MZ and JS wrote this manuscript. All authors read and approved the final manuscript.

\section{Author details}

${ }^{1}$ College of Bioinformatics Science and Technology, Harbin Medical University, Harbin 150081, People's Republic of China. ${ }^{2}$ Department of Interventional Radiology, The Affiliated Tumor Hospital of Harbin Medical University, Harbin, Heilongjiang 150040, People's Republic of China. ${ }^{3}$ School of Life Sciences, Jilin University, Changchun 130012, People's Republic of China.

\section{Acknowledgements}

This work was supported by Industrial Technology Research and Development Project, Jilin Province (Grant No. 2013C014-3) and the National Natural Science Foundation of China (Grant No. 61403111). The authors thank the seven anonymous reviewers for their constructive comments and suggestions for improving the manuscript. 


\section{Compliance with ethical guidelines}

\section{Competing interests}

The authors declare that they have no competing interests.

Received: 2 March 2015 Accepted: 1 June 2015

Published online: 17 July 2015

\section{References}

1. Jemal A, Bray F, Center MM, Ferlay J, Ward E, Forman D (2011) Global cancer statistics. CA Cancer J Clin 61 (2):69-90

2. She J, Yang P, Hong Q, Bai C (2013) Lung cancer in China: challenges and interventions. Chest 143(4):1117-1126

3. Ding L, Getz G, Wheeler DA, Mardis ER, McLellan MD, Cibulskis K et al (2008) Somatic mutations affect key pathways in lung adenocarcinoma. Nature 455(7216):1069-1075

4. Cheetham SW, Gruhl F, Mattick JS, Dinger ME (2013) Long noncoding RNAs and the genetics of cancer. Br J Cancer 108(12):2419-2425

5. Ma L, Bajic VB, Zhang Z (2013) On the classification of long non-coding RNAs. RNA Biol 10(6):925-933

6. Fatica A, Bozzoni I (2014) Long non-coding RNAs: new players in cell differentiation and development. Nat Rev Genet 15(1):7-21. doi:10.1038/ nrg3606

7. Wang KX, Chang HY (2011) Molecular mechanisms of long noncoding RNAs. Mol Cell 43(6):904-914. doi:10.1038/hr.2015.26

8. Tay Y, Rinn J, Pandolfi PP (2014) The multilayered complexity of ceRNA crosstalk and competition. Nature 505(7483):344-352

9. Kornienko AE, Guenzl PM, Barlow DP, Pauler FM (2013) Gene regulation by the act of long non-coding RNA transcription. BMC Biol 11:59

10. Maass PG, Luft FC, Bahring S (2014) Long non-coding RNA in health and disease. J Mol Med 92(4):337-346. doi:10.1007/s00109-014-1131-8

11. Qiu MT, Hu JW, Yin R, Xu L (2013) Long noncoding RNA: an emerging paradigm of cancer research. Tumour Biol 34(2):613-620

12. Prensner JR, Chinnaiyan AM (2011) The emergence of IncRNAs in cancer biology. Cancer Discov 1(5):391-407

13. Tang JY, Lee JC, Chang YT, Hou MF, Huang HW, Liaw CC et al (2013) Long noncoding RNAs-related diseases, cancers, and drugs. Sci World J 2013:943539. doi:10.1155/2013/943539

14. Gupta RA, Shah N, Wang KC, Kim J, Horlings HM, Wong DJ et al (2010) Long non-coding RNA HOTAIR reprograms chromatin state to promote cancer metastasis. Nature 464(7291):1071-1076

15. Ge X, Chen Y, Liao X, Liu D, Li F, Ruan H et al (2013) Overexpression of long noncoding RNA PCAT-1 is a novel biomarker of poor prognosis in patients with colorectal cancer. Med Oncol 30(2):588

16. Hauptman N, Glavac D (2013) Long non-coding RNA in cancer. Int J Mol Sci 14(3):4655-4669

17. Zhang H, Chen Z, Wang X, Huang Z, He Z, Chen Y (2013) Long noncoding RNA: a new player in cancer. J Hematol Oncol 6:37

18. Zhang W, Huang C, Gong Z, Zhao Y, Tang K, Li X et al (2013) Expression of LINC00312, a long intergenic non-coding RNA, is negatively correlated with tumor size but positively correlated with lymph node metastasis in nasopharyngeal carcinoma. J Mol Histol 44(5):545-554

19. Li J, Chen Z, Tian L, Zhou C, He MY, Gao Y et al (2014) LncRNA profile study reveals a three-IncRNA signature associated with the survival of patients with oesophageal squamous cell carcinoma. Gut 63(11):1700-1710

20. Chen J, Wang R, Zhang K, Chen LB (2014) Long non-coding RNAs in nonsmall cell lung cancer as biomarkers and therapeutic targets. J Cell Mol Med 18(12):2425-2436. doi:10.1111/jcmm.12431

21. Ji P, Diederichs S, Wang W, Boing S, Metzger R, Schneider PM et al (2003) MALAT-1, a novel noncoding RNA, and thymosin beta4 predict metastasis and survival in early-stage non-small cell lung cancer. Oncogene 22(39):8031-8041

22. White NM, Cabanski CR, Silva-Fisher JM, Dang HX, Govindan R, Maher CA (2014) Transcriptome sequencing reveals altered long intergenic noncoding RNAs in lung cancer. Genome Biol 15(8):429

23. Nie FQ, Zhu Q, Xu TP, Zou YF, Xie M, Sun M et al (2014) Long non-coding RNA MVIH indicates a poor prognosis for non-small cell lung cancer and promotes cell proliferation and invasion. Tumour Biol 35(8):7587-7594. doi:10.1007/s13277-014-2009-7
24. Zhang XQ, Sun S, Lam KF, Kiang KM, Pu JK, Ho AS et al (2013) A long non-coding RNA signature in glioblastoma multiforme predicts survival. Neurobiol Dis 58:123-131

25. Hu Y, Chen HY, Yu CY, Xu J, Wang JL, Qian J et al (2014) A long non-coding RNA signature to improve prognosis prediction of colorectal cancer Oncotarget 5(8):2230-2242

26. Meng J, Li P, Zhang Q, Yang Z, Fu S (2014) A four-long non-coding RNA signature in predicting breast cancer survival. J Exp Clin Cancer Res 33(1):84

27. Zhao W, Luo J, Jiao S (2014) Comprehensive characterization of cancer subtype associated long non-coding RNAs and their clinical implications. Sci Rep 4:6591

28. Yang J, Lin J, Liu T, Chen T, Pan S, Huang W et al (2014) Analysis of IncRNA expression profiles in non-small cell lung cancers (NSCLC) and their clinical subtypes. Lung Cancer 85(2):110-115

29. Botling J, Edlund K, Lohr M, Hellwig B, Holmberg L, Lambe M et al (2013) Biomarker discovery in non-small cell lung cancer: integrating gene expression profiling, meta-analysis, and tissue microarray validation. Clin Cancer Res Off J Am Assoc Cancer Res 19(1):194-204. doi:10.1158/10780432.CCR-12-1139

30. Okayama H, Kohno T, Ishii Y, Shimada Y, Shiraishi K, Iwakawa R et a (2012) Identification of genes upregulated in ALK-positive and EGFR/ KRAS/ALK-negative lung adenocarcinomas. Cancer Res 72(1):100-111. doi:10.1158/0008-5472.CAN-11-1403

31. Der SD, Sykes J, Pintilie M, Zhu CQ, Strumpf D, Liu N et al (2014) Validation of a histology-independent prognostic gene signature for early-stage, non-small-cell lung cancer including stage IA patients. Journal of thoracic oncology : official publication of the International Association for the Study of Lung Cancer. 9(1):59-64. doi:10.1097/JTO.0000000000000042

32. Irizarry RA, Bolstad BM, Collin F, Cope LM, Hobbs B, Speed TP (2003) Summaries of Affymetrix GeneChip probe level data. Nucleic Acids Res 31(4):e15

33. Irizarry RA, Hobbs B, Collin F, Beazer-Barclay YD, Antonellis KJ, Scherf U et al (2003) Exploration, normalization, and summaries of high density oligonucleotide array probe level data. Biostatistics. 4(2):249-264

34. Harrow J, Frankish A, Gonzalez JM, Tapanari E, Diekhans M, Kokocinski F et al (2012) GENCODE: the reference human genome annotation for The ENCODE Project. Genome Res 22(9):1760-1774. doi:10.1101/ gr.135350.111

35. Zhang X, Sun S, Pu JK, Tsang AC, Lee D, Man VO et al (2012) Long noncoding RNA expression profiles predict clinical phenotypes in glioma. Neurobiol Dis 48(1):1-8

36. Lossos IS, Czerwinski DK, Alizadeh AA, Wechser MA, Tibshirani R, Botstein D et al (2004) Prediction of survival in diffuse large-B-cell lymphoma based on the expression of six genes. N Engl J Med 350(18):1828-1837

37. da Huang W, Sherman BT, Lempicki RA (2009) Bioinformatics enrichment tools: paths toward the comprehensive functional analysis of large gene lists. Nucleic Acids Res 37(1):1-13

38. Sanchez-Palencia A, Gomez-Morales M, Gomez-Capilla JA, Pedraza V, Boyero L, Rosell R et al (2011) Gene expression profiling reveals novel biomarkers in nonsmall cell lung cancer. Int J Cancer 129(2):355-364. doi:10.1002/ijc.25704

39. Guo NL, Wan YW (2012) Pathway-based identification of a smoking associated 6-gene signature predictive of lung cancer risk and survival. Artif Intell Med 55(2):97-105

40. Heagerty PJ, Lumley T, Pepe MS (2000) Time-dependent ROC curves for censored survival data and a diagnostic marker. Biometrics. 56(2):337-344

41. Merico D, Isserlin R, Stueker O, Emili A, Bader GD (2010) Enrichment map: a network-based method for gene-set enrichment visualization and interpretation. PLoS One 5(11):e13984. doi:10.1371/journal.pone.0013984

42. Shannon P, Markiel A, Ozier O, Baliga NS, Wang JT, Ramage D et al (2003) Cytoscape: a software environment for integrated models of biomolecular interaction networks. Genome Res 13(11):2498-2504. doi:10.1101/ gr.1239303

43. Kratz JR, Jablons DM (2009) Genomic prognostic models in early-stage lung cancer. Clin Lung Cancer. 10(3):151-157

44. Zhu CQ, Pintilie M, John T, Strumpf D, Shepherd FA, Der SD et al (2009) Understanding prognostic gene expression signatures in lung cancer. Clin Lung Cancer. 10(5):331-340 
45. Gibb EA, Vucic EA, Enfield KS, Stewart GL, Lonergan KM, Kennett JY et al (2011) Human cancer long non-coding RNA transcriptomes. PLoS One 6(10):e25915

46. Su X, Malouf GG, Chen Y, Zhang J, Yao H, Valero V et al (2014) Comprehensive analysis of long non-coding RNAs in human breast cancer clinical subtypes. Oncotarget. 5(20):9864-9876

47. Du Z, Fei T, Verhaak RG, Su Z, Zhang Y, Brown M et al (2013) Integrative genomic analyses reveal clinically relevant long noncoding RNAs in human cancer. Nat Struct Mol Biol 20(7):908-913

48. Subramanian J, Simon R (2010) Gene expression-based prognostic signatures in lung cancer: ready for clinical use? J Natl Cancer Inst 102(7):464-474

49. Skarin AT, Herbst RS, Leong TL, Bailey A, Sugarbaker D (2001) Lung cancer in patients under age 40. Lung Cancer 32(3):255-264
50. Hsu CL, Chen KY, Shih JY, Ho CC, Yang CH, Yu CJ et al (2012) Advanced non-small cell lung cancer in patients aged 45 years or younger: outcomes and prognostic factors. BMC Cancer 12:241

51. Chen KY, Chang CH, Yu CJ, Kuo SH, Yang PC (2005) Distribution according to histologic type and outcome by gender and age group in Taiwanese patients with lung carcinoma. Cancer 103(12):2566-2574

52. Kalemkerian GP, Akerley W, Bogner P, Borghaei H, Chow LQ, Downey RJ et al (2013) Small cell lung cancer. J Natl Compr Canc Netw 11(1):78-98

53. Hangauer MJ, Vaughn IW, McManus MT (2013) Pervasive transcription of the human genome produces thousands of previously unidentified long intergenic noncoding RNAs. PLoS Genet 9(6):e1003569
Submit your next manuscript to BioMed Central and take full advantage of:

- Convenient online submission

- Thorough peer review

- No space constraints or color figure charges

- Immediate publication on acceptance

- Inclusion in PubMed, CAS, Scopus and Google Scholar

- Research which is freely available for redistribution

Submit your manuscript at www.biomedcentral.com/submit 